\title{
On Normalized Convolution to Measure Curvature Features for Automatic Polyp Detection
}

\author{
C. van Wijk ${ }^{1}$, R.Truyen ${ }^{2}$, R. E. van Gelder ${ }^{3}$, L.J. van Vliet $^{1}$, and F.M. Vos ${ }^{1,3}$ \\ 1 Quantitative Imaging Group, Delft University of Technology \\ Lorentzweg 1, 2628 CJ Delft, The Netherlands \\ \{vanwijk, lucas, frans\}@ph.tn.tudelft.nl \\ 2 MIMIT AD Group, Philips Medical Systems Nederland B.V. \\ P.O. Box 10000, 5680 DA Best, The Netherlands \\ \{roel.truyen\}@philips.com \\ 3 Department of Radiology, Academic Medical Centre Amsterdam \\ P.O. Box 22700, 1100 DE Amsterdam, The Netherlands \\ $\{$ r.e.vangelder, f.m.vos\}@amc.uva.nl
}

\begin{abstract}
Early removal of polyps has proven to decrease the incidence of colon cancer. We aim to increase the sensitivity of the screening by automatic detection of polyps. It requires accurate measurement of the colon wall curvature. This paper describes a new method which computes the curvatures using space-variant derivative operators in a strip along the edge of the colon. It optimizes the trade-off between noise reduction and mixing of adjacent image structures. The derivative operators incorporate an applicability function for regularization and interpret the strips as confidence measure; certain inside and uncertain outside. To that purpose the technique of normalized convolution is utilized and adapted to allow a local Taylor expansion of the image signal. A special scheme to compute the confidence values is also presented.
\end{abstract}

\section{Introduction}

The colorectal polyp is an important precursor to colon cancer [1013]. This benign lesion typically protrudes from the colon wall as a small, sloped mound (see Figure 1). Fortunately, the long pre-malignant stadium (5-10 years) enables efficient prevention by a timely removal. Virtual colonoscopy is a procedure to inspect the colon based on 3D CT images. However, current visualization techniques are still too time consuming for large scale use. Additionally, significant polyps are sometimes missed. Therefore many authors have suggested methods of automatic polyp detection [8,54,6].

Accurate curvature measurement is essential for any polyp detection scheme. Yoshida reported a method based on the implicit function theorem, computing 

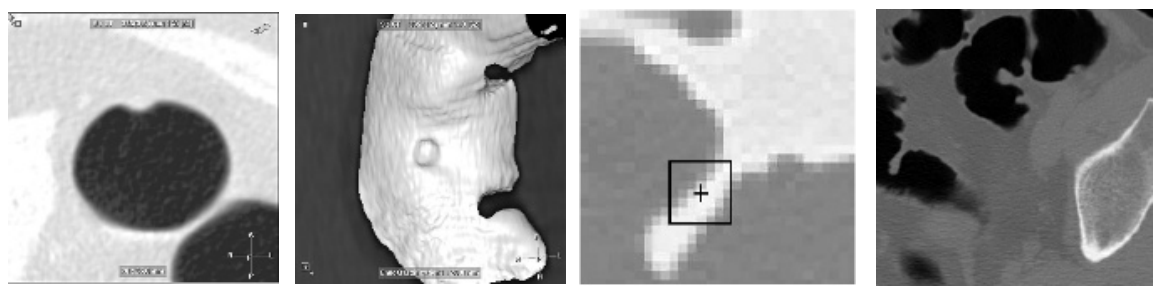

Fig. 1. From left to right: First two pictures: Cross-sectional view of small polyp and endoluminal view of same polyp. The last two pictures show a thin fold with overlay of a $7 \times 7$ kernel and tissue and folded structures

the radius of curvature directly from the first and second order derivatives. These derivatives were computed by employing isotropic Gaussian derivative kernels at scale $\sigma[15]$. Summers advocates a local B-spline fit through the triangulated surface voxels from which the radius of curvature is calculated 11. Unfortunately a local model obtained from only surface voxels (without using grey value information) tends to become rather noisy. We have seen this happening in the computation of surface normals from triangulated meshes [9].

Noisy data asks for a certain amount of regularization, whereas thin colonic structures require a very small filter kernel. Violating the first requirement yields a noisy result (stochastic error) whereas violating the second causes a substantial bias in the derivatives (systematic error). Both errors hamper the curvature measurement. Finding a trade-off between the conflicting requirements is very difficult due to the presence of small folded structures on the colon wall as well as the presence of other structures in the tissue (see Figure 10.

In this paper we present a novel method to adapt the size and shape of the filter kernels to the local image data. The method avoids the systematical error due to mixing of nearby image structures and is optimized for noise reduction. However, using irregular shaped filter kernels requires a space-variant normalization of the derivative filters. Therefore we present an intuitive framework for deriving normalized differential convolution of arbitrary order (Section 2.1). In section 2.2 we present a scheme to compute space-variant kernels from the local image structure. The performance of the new method is assessed on both simulated data as well as CT data.

\section{Methods}

Derivatives in 3D images can be computed by convolution with derivatives of Gaussian kernels. In order to adapt the Gaussian (derivative) kernels to the local geometry they are multiplied with a confidence function which is extracted from

\footnotetext{
${ }^{1}$ Unfortunately no information on the scale at which the derivatives are computed is given.
} 
the local image structure. This additional weighting requires re-normalization as well as a (re-)orthogonalization. The technique which takes care of both is normalized convolution ( $[7 \mid 14,3,2])$.

For the detection of polyps the resulting image derivatives can be combined into principal curvatures, $\kappa_{1}$ and $\kappa_{2}$ (Thirion and Gourdon [12]). Based on the principal curvatures a number of polyp detectors can be constructed. Yoshida [15] uses primarily the shape index and curvedness. The shape index is given by $S I=\frac{1}{2}-\frac{1}{\pi} \operatorname{atan}\left(\frac{\kappa_{1}+\kappa_{2}}{\kappa_{1}-\kappa_{2}}\right)$ and the curvedness is given by $C V=\sqrt{\frac{k_{1}^{2}+k_{2}^{2}}{2}}$.

\subsection{A Least Squares Approach to Normalized Convolution}

The following assumes a 2D image (extension to 3D image space is straightforward). Consider a local neighbourhood of $N \times N$ pixels $f_{i}$ that is modeled by a Taylor expansion around the center of the local neighborhood (indicated by 0 ):

$$
f_{i}=I(0)+x_{i} I_{x}(0)+y_{i} I_{y}(0)+\frac{x_{i}^{2} I_{x x}(0)}{2 !}+\frac{y_{i}^{2} I_{y y}(0)}{2 !}+\frac{2 x_{i} y_{i} I_{x y}(0)}{2 !}+R(i)
$$

in which $I$ indicates the 'true', underlying image function and $i$ is a linear index. Using terms up to the second order and substituting $\eta_{1}=I(0), \eta_{2}=I_{x}(0), \ldots$, Equation 1 is rewritten as:

$$
\left[\begin{array}{c}
f_{1} \\
\cdots \\
f_{N^{2}}
\end{array}\right] \approx\left[\begin{array}{cccccc}
1 & x_{1} & y_{1} & 0.5 x_{1}^{2} & 0.5 y_{1}^{2} & x_{1} y_{1} \\
\ldots & \cdots & \cdots & \cdots & \cdots & \cdots \\
1 & x_{N^{2}} & y_{N^{2}} & 0.5 x_{N^{2}}^{2} & 0.5 y_{N^{2}}^{2} & x_{N^{2}} y_{N^{2}}
\end{array}\right]\left[\begin{array}{c}
\eta_{1} \\
\cdots \\
\eta_{6}
\end{array}\right]
$$

The local neighbourhood can be depicted as a point in an $N^{2}$-dimensional space spanned by the orthonormal basis $\left\{\mathbf{e}_{i}\right\}$. A new set of basis vectors $\mathbf{b}_{j}=\left\{\mathbf{1}, \mathbf{x}, \mathbf{y}, \frac{\mathbf{x x}}{2}, \frac{\mathbf{y y}}{\mathbf{2}}, \mathbf{x y}\right\}$ are the basis functions of the Taylor expansion (i.e. the columns of the matrix in Equation 2). Thus, $\left\{\eta_{1}, \eta_{2}, \eta_{3}, \eta_{4}, \eta_{5}, \eta_{6}\right\}$ are the coordinates of the signal on the new basis and directly yield the first and second order derivatives. It can be stated that:

$$
f_{e}^{i} \approx \mathbf{B} \eta_{b}^{j}
$$

Equation 3 merely rewrites Equation 2 implying that the signal $f$ on basis $\mathbf{e}_{i}$ is approximated by the so-called basis tensor $\mathbf{B}$ times the coordinates of $f$ on basis $\mathbf{b}_{j},\left(\eta_{b}^{j}\right)$. It must be emphasized that, in general, the basis functions can be freely selected and need not be orthonormal. Our basis was merely chosen to comply with the Taylor expansion. The objective now is to find the new coordinates $\eta_{b}^{j}$ by minimizing the error $\epsilon=\|f-\mathbf{B} \eta\|=(f-\mathbf{B} \eta)^{2}$. The result is the general least squares solution to 3 .

$$
\left(\mathbf{B}^{T} \mathbf{B}\right)^{-1} \mathbf{B}^{T} f_{e}^{i}=\eta_{b}^{j}
$$

with $\left(\mathbf{B}^{T} \mathbf{B}\right)^{-1} \mathbf{B}^{T}$ the pseudo-inverse of $\mathbf{B}$. 
To reduce the influence of points further away from the neighborhood center we multiply the set of equations in eq. 2 by a (rotation invariant) matrix $\hat{\mathbf{A}}$, with $\mathbf{A}=\hat{\mathbf{A}}^{T} \hat{\mathbf{A}}$. The $N^{2} \times N^{2}$ diagonal matrix $\mathbf{A}$ contains the spatial weights and is called the applicability function 2 .

$$
\hat{\mathbf{A}} f_{e}^{i}=\hat{\mathbf{A}} \mathbf{B} \eta_{b}^{j}
$$

Multiplication with $\hat{\mathbf{A}}$ is allowed as long as it does not yield a singular system of equations. Similarly, each equation in (2) can be multiplied again by other weights. It is now clear how confidence levels assigned to each neighbor can be incorporated. The result is a double weighted least squares solution:

$$
\left(\mathbf{B}^{T} \mathbf{A C B}\right)^{-1} \mathbf{B}^{T} \mathbf{A C} f_{e}^{i}=\eta_{b}^{j}
$$

with the diagonal matrix $\mathbf{C}=\hat{\mathbf{C}}^{T} \hat{\mathbf{C}}$ holding the confidence value of each neighbor.

\subsection{Local Confidence Values}

The framework presented in the previous section accommodates normalized space variant-kernels. The confidence values which are inserted into this regularization are computed locally and will adapt the kernel to the local image structure. The goal is to assign high confidence to voxels on the image structure under consideration and a low confidence to neighboring structures. Such structures might be neighboring folds, changes in tissue structures, the opposite side of a fold, etc. We propose the following scheme to compute the confidence values.

1. Segment the air to find the air-tissue interface. Usually this is achieved by simple thresholding. We use a dynamic threshold [1] to allow for a correct segmentation of geometries affected by partial volume effects.

2. Compute for all voxels the distance to the air-tissue interface. We perform two distance transforms. One to compute the distance to air. From this we subtract a second distance transform, the distance to tissue. This operation results in positive values for air and negative values for tissue. On the colon wall the values of the distance transform are zero.

3. Compute the gradient of the distance transform which will act as a normal vector field. We will use these normals to distinct between different structures.

Steps 1 to 3 can be computed for the entire image at once. In contrast the following step is a local one to be incorporated in the convolution process. To distinguish between different geometries one can remark that the surface normal of the structure under consideration will differ from that of the direct neighboring structures.

${ }^{2}$ We use a Gaussian weighting $A=e^{-\frac{x^{2}}{2 \sigma^{2}}}$ such that the scale at which the least squares solution is obtained is identical to the scale at which isotropic Gaussian derivatives are computed. 

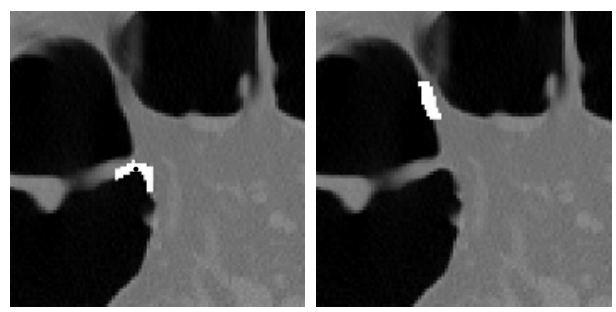

Fig. 2. Constructed confidence values for a neighborhood of $13^{2}$ pixels. The neighborhood center is indicated by the black dot.

4. Assign neighboring voxels to belong to the current structure by taking the inner product of the normal at the neighborhood center with the normal of a neighbor. A threshold on this value (e.g. ¿0) classifies the neighbor and sets it's confidence value to zero or one.

An example of a region selected by the above scheme is given in Figure 2 . Note that the confidence values are weighted with the applicability function in the regularization process.

\section{Results}

The performance of the space-variant filtering is assessed on both simulated objects as well as CT data. Two test images were created to test the computation of radii of curvature with both the isotropic method as well as the new method. The first image, displayed in Figure $3 \mathrm{a}$, is a 3D cylinder (only cross-section shown) which was constructed using the error function with a $\sigma$ of 2 . The cylinder has a radius of 18 pixels. Gaussian noise was added to the images. The standard deviation of the noise was $4 \%$ of the contrast (intensity difference between air and tissue). The second test image contains two 3D cylinders, their centers separated by 40 pixels. The image was constructed by multiplying two separate cylinder images after which noise was added.

Figure 3 shows that noise affects the derivative computation at small scales (a and b). Increasing the (isotropic) scale of the operator improves the results (c), but adjacent structures inside the footprint of the filter spoil the final result (d).

The isotropic Gaussian derivative filtering fails to return the correct curvatures. In this paper we propose to improve the curvature measurement by introducing space variant kernels. The performance is compared to the isotropic method in Figure 4. The result of the isotropic method are repeated on the left cylinder. The results obtained with the proposed method are plotted on the right cylinder. It is clear that in the region where the two cylinders are close together the new method outperforms the method using isotropic kernels. 

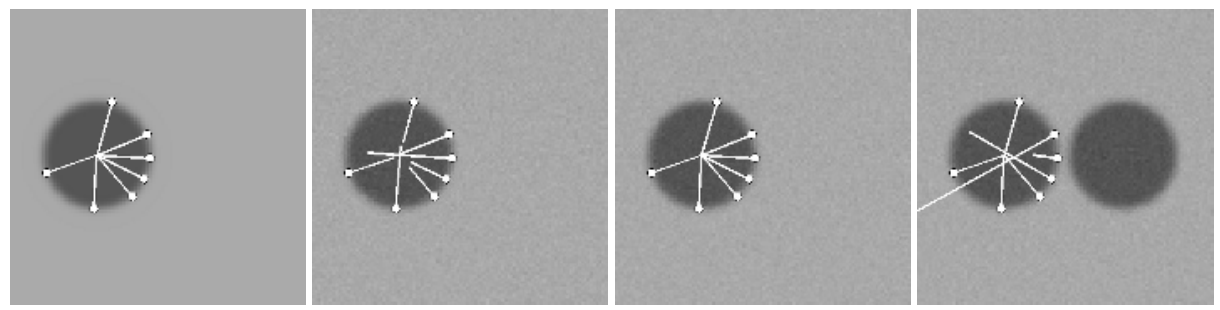

Fig. 3. Trade-off between noise suppression and resolution. On several positions on the edge the normal direction (line direction) and radius of curvature (line length) are plotted. From left to right: (a) noise free image, small scale $\sigma=1$. (b) Gaussian noise added, $\sigma=1$. (c) computation at larger scale suppresses the noise, $\sigma=3$. At larger scale $(\sigma=3)$ incorrect curvature and gradient direction are obtained close to neighboring structures $(d)$.
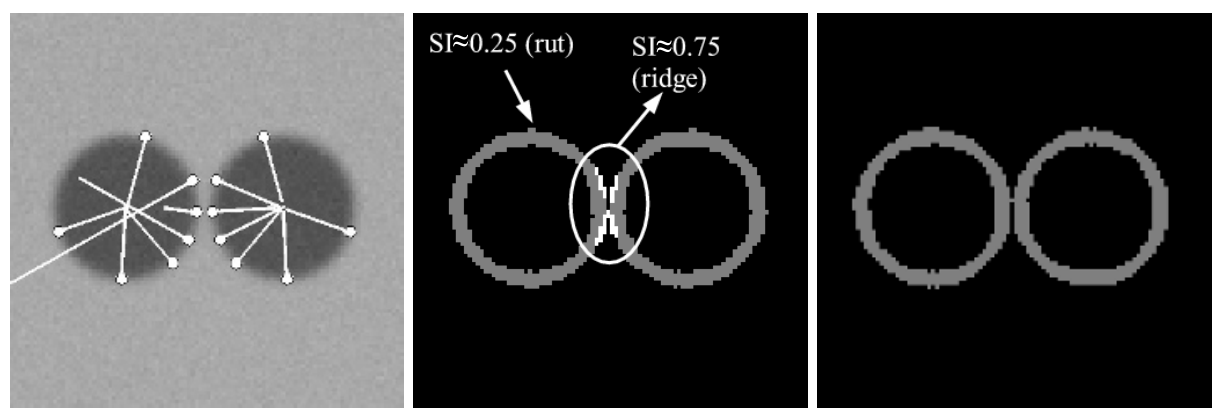

Fig. 4. Radius of curvature (left) and shape index (middle,right) computed on two cylinders (Only a cross section of the cylinders is shown). On several positions on the edge the gradient (line direction) and radius of curvature (line length) are plotted. Left cylinder: isotropic method. The gradient direction is obtained using isotropic Gaussian kernels $(\sigma=3)$. Right cylinder: both gradient direction and radius of curvature are obtained with space variant kernels $(\sigma=3)$.

The middle image shows the classification by shape index computed by the isotropic method. The isotropic method classifies large part of the cylinders to a ridge like structure. The new method (right) using space variant kernels classifies all voxels correctly.

The new method does not suffer from the systematic error introduced when using isotropic filters. The cost is a small increase in a stochastic error due to the fact that the incorporation of confidence levels into the filtering in effect reduces the number of voxels used to suppress noise. However, the specific choice of confidence levels based on the local structure allows to discard just those voxels which would have introduced a systematic error. In other words our method optimizes the trade-off between noise reduction and preservation of image structure.

The shape index is computed from the principal curvatures and is often used to select polyp candidates by means of thresholding. Applying such classification to the image in Figure 4a yield Figure 4b. The isotropic method will result in 

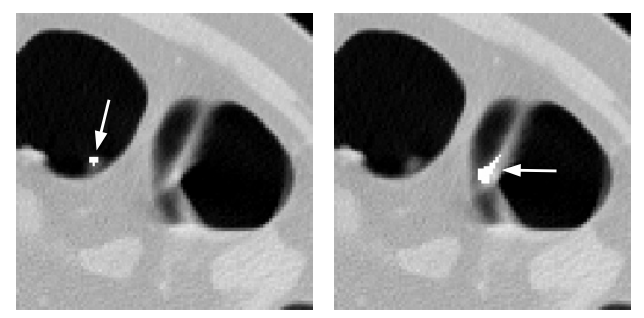

Fig. 5. One slice from the 3D Ct dataset. Voxels labelled as belonging to polyps (white). The new method finds the polyp (left). The isotropic method fails to find the polyp and selects a false positive. The results were obtained by filtering with $\sigma=3$.

Table 1. Detection results. The isotropic method detects 3 false positives. The space variant method detection the true positive and one (small) false positive. The results were obtained by filtering with $\sigma=3$.

\begin{tabular}{|c|c|c|c|}
\hline id & method & cluster size & label \\
\hline 1 & isotropic & 9 & false positive \\
\hline 2 & isotropic & 28 & false positive \\
\hline 3 & isotropic & 86 & false positive \\
\hline 4 & space-variant & 31 & true positive \\
\hline 5 & space-variant & 3 & false positive \\
\hline
\end{tabular}

a classification of a large part of the cylinder to a ridge-like structure. The new method correctly classifies all the voxels to a rut-like structure (8c).

To demonstrate the performance of the method on CT data, a scheme similar to 8 is applied. Yoshida et al. use the the shape index and curvedness to select the set of polyp candidates. In [8] thresholds were presented for the shape index (between 0.9 and 1.0) and for curvedness $\left(0.05 \mathrm{~mm}^{-1}\right.$ and $\left.0.25 \mathrm{~mm}^{-1}\right)$. We applied the same scheme using hysteresis thresholding [8] to investigate the performance with respect to the candidate selection step. Initial test results on a few patients show promising results. The power of the method is clearly demonstrated in Figure 5 and Table 10 which are obtained by applying the method to a small dataset $(200 \times 200 \times 100$ voxels $)$ containing one polyp (approx. $4 \mathrm{~mm}$ ). The new method detects the polyp and finds one false positive. The isotropic method detects three false positives and misses the true positive.

From the demonstration of our method both on simulated data as well as CT data we feel confident that space-variant kernels will yield fewer false positives. Especially for small polyps the new method is likely to increase the sensitivity. However, we are aware that the performance of the operator can only be assessed by statistical validation on a large number of datasets. This is the focus of future work. 


\section{Conclusions}

The measurement of curvature in CT data for the detection of polyps is difficult due to the highly folded colon. Therefore noise suppression with larger isotropic filters is not possible. We have shown that with a specific formulation of normalized convolution using a local Taylor expansion space-variant kernels can be used. In addition we have shown that space-variant kernels can be constructed which discards just those voxels belonging to neighboring image geometries. Thereby the derivative filtering optimizes the trade-off between noise suppression and preservation of local image structure.

The assessment of the method by simulated images shows that the space-variant filtering outperforms isotropic filtering. Also, on CT data the new method seems to indicate a higher sensitivity and higher specificity. However, the authors do realize an investigation on more data is needed to be conclusive on the overall improvement of polyp detection.

\section{References}

1. J. Bernsen: Dynamic Thresholding of grey level images. In ICPRA'86: Proc. Int. Conf. on Pattern Recognition, pp 1251-1255, Berlin, Germany, 1986

2. G. Farnebäck: A Unified Framework for Bases, Frames, Subspace Bases, and Subspace Frames. SCIA 1999, pages 341-349, Kangerlussuaq, Greenland, 1999.

3. Gunnar Farnebäck. Spatial Domain Methods for Orientation and Velocity Estimation. PhD Thesis, Linköping University, Sweden, 1999.

4. S.B. Goturk et al.: A statistical 3-d pattern processing method for computer-aided detection of polyps in ct colonography. IEEE Trans. on Med. Imag. 20:1251-1260, 2001

5. A. K. Jerebko et al.: Multiple neural network classification scheme for detection of colonic polyps in ct colonography data sets. Acad. Radiol., 10:154-160, 2003

6. G. Kiss et al.: Computer-aided diagnosis in virtual colonography via combination of surface normal and sphere fitting methods. European Radiology, 12:77-88, 2002

7. H. Knutsson and C.-F. Westin: Normalized Convolution - a technique for filtering incomplete and uncertain data. SCIA, 2:997-1006, 1993

8. J. Nappi and H. Yoshida: Automated detection of polyps with ct colonography: evaluation of volumetric features for reduction of false-positive findings. Acad. Radiol., 9:386-397, 2002

9. M. Persoon, I.W.O. Serlie, F. Post, R. Truyen and F. M. Vos: Visualization of noisy and biased volume data using first and second order derivative techniques. IEEE Visualization 2003 (Proc. Conf. Seattle, WA), pages 379-385, 2003

10. J.D. Potter, M.L. Slattery and R. M. Bostick. Colon cancer: a review of the epidemiology. Epidemiol. Rev., 15:499-545, 1993

11. R. M. Summers, C. F. Beaulieu, L. M. Pusanik, J. D. Malley, R. Brooke, J. D. Glazer and S. Napel: Automated polyp detector for ct colonography: Feasibility Study. Radiology, 216:284-290, 2000

12. J.P. Thirion and A. Gourdon. Computing the Differential Characteristics of Isointensity Surfaces. Computer Vision and Image Understanding, 61(2):190-202, 1995 
13. B. Vogelstein, E. R. Fearon and S. R. Hamilton: Genetic alterations during colorectal-tumour development. N.Eng.J.Med., 319:525-532, 1998

14. C-.F. Westin A Tensor Framework for Multidimensional Signal Processing. PhD Thesis, Linköping University, Sweden, 1994.

15. H. Yoshida: Three-dimensional computer-aided diagnosis scheme for detection of colonic polyps. IEEE Transactions on Medical Imaging, 20:1261-1274, 2001 